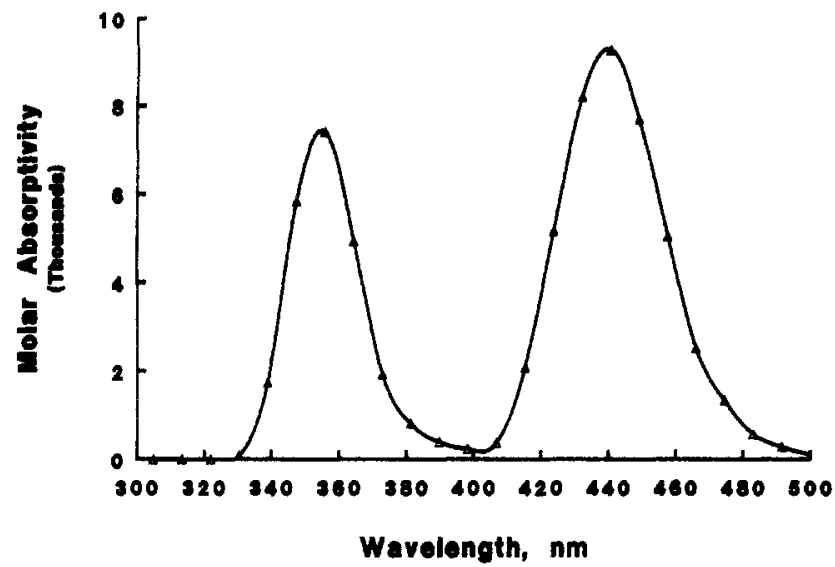

Figure 9. Calculated charge-transfer spectrum of indene-TCNE at 77 $\mathrm{K}$, averaged over donor-acceptor distance $z$.

The resulting approximation to the line shape is shown in Figure 8 for a temperature of $25^{\circ} \mathrm{C}$. This spectrum can be compared to the experimental solution phase spectra of Figure 1, which are additionally influenced by the tilting motion of the molecular planes as well as the inhomogeneous broadening of the solvent The calculated maxima in Figure 8 are at 356 and $449 \mathrm{~nm}$, and the corresponding calculated molar absorptivities are 5260 and $6530 \mathrm{M}^{-1} \mathrm{~cm}^{-1}$.

The theoretical $R$-averaged spectrum was also calculated at temperatures of 5 and $35^{\circ} \mathrm{C}$, but the resulting line shapes were not very different from that shown in Figure 9. A slight broadening with increasing temperature leads to a decrease in the maximum molar absorptivity with temperature. The calculated molar absorptivity at $440 \mathrm{~nm}$ varies from 8940 to $5280 \mathrm{M}^{-1} \mathrm{~cm}^{-1}$ on going from 5 to $35^{\circ} \mathrm{C}$.

The spectrum calculated at $77 \mathrm{~K}$, shown in Figure 8, is considerably sharper than the room temperature spectrum, in agreement with experiments on related system. ${ }^{4}$ The calculated molar absorptivities at 356 and $440 \mathrm{~nm}$ are 7414 and $9270 \mathrm{M}^{-1}$ $\mathrm{cm}^{-1}$, respectively.

\section{Conclusions}

In this work we have explored the orientation dependence of spectroscopic electron transfer in the indene-TCNE EDA complex. By calculating the energy and CT spectra as a function of complex geometry, it has been shown that the first and second chargetransfer transitions are allowed in a wide range of energetically accessible conformations. Investigation of the ground-state intermolecular potential also shows that fairly small energy barriers of the order of $k T$ at room temperature separate the minima associated with possible orientational and translational isomers. Thus, for indene-TCNE, there is no correspondence between the two CT bands and distinct complex geometries.

Acknowledgment. W.D.E. acknowledges a seed grant from the University of Idaho Research Council and generous start-up funds needed to acquire the components of The Computational Facility for Theoretical Chemistry. W.D.E. and J.L.M. thank the National Science Foundation's EPSCoR program for grant R11-8902065. J.L.M. acknowledges the support of NSF grant 8605079 .

\title{
AM1 and INDO/S Calculations on Electronic Singlet and Triplet States Involved In Excited-State Intramolecular Proton Transfer of 3-Hydroxyflavone
}

\author{
Bernhard Dick \\ Max-Planck-Institut für biophysikalische Chemie, Abteilung Laserphysik, D-3400 Göttingen, \\ Federal Republic of Germany (Received: November 21, 1989)
}

\begin{abstract}
Energies and geometries of the electronic ground states and vertical transition energies and oscillator strengths of singlet-singlet and triplet-triplet transitions have been calculated for both tautomer forms of 3-hydroxyflavone. The resulting energy level diagram and calculated excited-state spectra yield a consistent interpretation of the data from transient absorption measurements. It is concluded that all long-lived transient absorptions are due to triplet species and that an intrinsic barrier to proton transfer exists in the lowest triplet state.
\end{abstract}

\section{Introduction}

Among the molecules which display an excited-state intramolecular proton transfer (ESIPT) reaction, 3-hydroxyflavone (3-HF) is one of the most extensively studied. However, as more and more experimental information on this system is collected, it appears that a simple four-level picture involving the ground states and the first excited singlet states of the normal form and the tautomer form of this molecule cannot explain all the observations. In particular, the observation of long-lived transient absorptions ${ }^{1-4}$ has stimulated hope to find an intrinsic barrier to the backward (ground-state) intramolecular-proton-transfer reaction, but the tautomer ground state could not be trapped at low temperatures.

(1) Itoh, M.; Fujiwara, Y, J. Phys. Chem. 1983, 87, 4558.

(2) Itoh, M.; Tanimoto, Y.; Tokumura, K. J. Am. Chem. Soc. 1983, 105, 3339.

(3) Itoh, M.; Fujiwara, Y.; Sumitani, M.; Yoshihara, K. J. Phys. Chem 1986, 90,5672 .

(4) Brewer, E. W.; Studer, S. L.; Standiford, M.; Chou, P.-T. J. Phys. Chem. 1989, 93, 6088 .
An impediment to the interpretation of the experimental data is the fact that the relative ordering of the singlet and triplet states of both tautomer forms of $3-\mathrm{HF}$ is not known. Bouman et al. ${ }^{5}$ have performed $a b$ initio calculations on the ground state and the first $\pi \pi^{*}$ excited state of $3-\mathrm{HF}$, but calculations on other states do not yet exist. This paper presents results of semiempirical molecular-orbital calculations for several singlet and triplet levels of both tautomer forms of 3-HF. For the triplet manifold of the zwitterionic tautomer these calculations predict rather unusual properties which yield a consistent interpretation of the experimental data available so far.

These experimental findings will be briefly summarized in the following. We denote the singlet and triplet states of the normal form of the molecule by the symbols $S_{n}$ and $T_{n}$ and the corresponding states of the tautomer form by $S_{n}{ }^{\prime}$ and $T_{n}{ }^{\prime}$. (In other molecules these forms are often termed enol and keto forms. In 3-HF, however, proton transfer changes the enol form to a 4460

(5) Bouman. T. D.; Knobeloch, M. A.; Bohan, S. J. Phys. Chem. 1985, 89, 
zwitterionic enolate structure for which no electrically neutral keto structure can be drawn.)

Excitation of the normal form of 3-HF leads to the first excited singlet state $S_{1}$ of $\pi \pi^{*}$ character. The first maximum of this transition occurs at $30800 \mathrm{~cm}^{-1}$ in the gas phase at $478 \mathrm{~K}^{6}$ and at $28400 \mathrm{~cm}^{-1}$ in alkane solution at room temperature. ${ }^{7}$ The extinction coefficient is $1.7 \times 10^{4} \mathrm{M}^{-1} \mathrm{~cm}^{-1}$ at the maximum of the first absorption band at $29200 \mathrm{~cm}^{-1} .^{8}$ Via rapid proton transfer $S_{1} \rightarrow \rightarrow S_{1}{ }^{\prime}$ population is transferred to the first excited singlet state of the tautomer. The fluorescence $S_{1}{ }^{\prime} \rightarrow S_{0}{ }^{\prime}$ of this species in alkane solutions peaks at $\lambda=526 \mathrm{~nm}\left(19000 \mathrm{~cm}^{-1}\right)$ with a quantum yield of 0.36 and a decay time of $3 \mathrm{~ns}$ at room temperature in methylcyclohexane $(\mathrm{MCH}),{ }^{1,9}$ Other papers report a decay time of $3 \mathrm{~ns}$ in benzene, ${ }^{1} 4 \mathrm{~ns}$ in cyclohexane, ${ }^{10}$ and $4.6 \mathrm{~ns}$ in $\mathrm{MCH} .11$ When the molecule is isolated in an argon matrix at a temperature of $15 \mathrm{~K}$, the ESIPT reaction is still very fast (unresolved with a time resolution of $2 \mathrm{ps}^{12}$ ) and apparently not hindered by an intrinsic barrier. The decay time of the tautomer fluorescence increases to $8 \mathrm{~ns}$ under these conditions. The origin of the $S_{0} \rightarrow S_{1}$ transition of the isolated molecule in a supersonic jet has a Lorentzian line shape with a homogeneous line width of $4.1 \mathrm{~cm}^{-1}, 13$ which may be interpreted in terms of a lifetime of 1.3 ps for the $S_{1}$ state.

Transient absorption measurements of 3-HF in hydrocarbon solvents have been performed in the picosecond ${ }^{10,14}$ and microsecond ${ }^{1-4}$ time domain. (Investigations performed with $3-\mathrm{HF}$ in hydrogen-bonding solvents ${ }^{8,11,15-17}$ like alcohols and ethers are not considered here, since the hydrogen-bonded complexes of 3-HF with solvent molecules behave distinctly differently from the uncomplexed molecule. ${ }^{18,19}$ Immediately after excitation, gain due to stimulated emission at $18500 \mathrm{~cm}^{-1}$ and a transient absorption at $16500 \mathrm{~cm}^{-1}$ are observed. ${ }^{14}$ Both transients rise very fast (unresolved with a time resolution of $30 \mathrm{ps)}$ ) and decay with the same time constant as the tautomer fluorescence. They can be assigned to transitions $S_{1}{ }^{\prime} \rightarrow S_{0}{ }^{\prime}$ and $S_{1}{ }^{\prime} \rightarrow S_{n}{ }^{\prime}$. Another transient absorption at $22700 \mathrm{~cm}^{-1}$ with $4 \mathrm{~ns}$ decay time has been reported in ref 10 . Two transient absorptions with decay times of several microseconds have been observed in non-hydrogenbonding solvents: ${ }^{1-4}$ The first has a peak at $395 \mathrm{~nm}\left(25300 \mathrm{~cm}^{-1}\right)$ and decays with a time constant of $7.2 \mu \mathrm{s}$. It is quenched by oxygen with a bimolecular rate constant of $2.1 \times 10^{9} \mathrm{M}^{-1} \mathrm{~s}^{-1}$, and singlet oxygen is formed. The second transient absorption peaks at $437 \mathrm{~nm}\left(22900 \mathrm{~cm}^{-1}\right)$ and decays with a time constant of 14.2 $\mu \mathrm{s}$. It is also quenched by oxygen with a slightly higher bimolecular rate constant of $3.2 \times 10^{9} \mathrm{M}^{-1} \mathrm{~s}^{-1}$. (In ref 2 the decay time of this transient was reported to be $2.9 \mu \mathrm{s}$ in aerated solution, which did not change much upon passing a stream of nitrogen through the sample. However, the same group found that in benzene solution the lifetime of this transient increases from 5.9 to $14.5 \mu$ s when the sample was deaerated. ${ }^{1}$ ) Excitation of this transient species with a second light pulse results in fluorescence from the tautomer, $S_{1}{ }^{\prime} \rightarrow S_{0}{ }^{\prime 1,3,4}$ but with a quantum yield of only 0.013 .4

On the basis of the calculations discussed below, the first transient absorption is assigned to $\mathrm{T}_{1} \rightarrow \mathrm{T}_{n}$ transitions and the second transient absorption to $T_{1}^{\prime} \rightarrow T_{n}{ }^{\prime}$ transitions. The first

(6) Itoh, M.: Kurokawa, H. Chem. Phys. Lett. 1982, 9l, 487.

(7) Sengupta, P. K.; Kasha, M. Chem. Phys. Lett. 1979, 68, 382.

(8) Strandjord, A. J. G.; Courtney, S. H.; Friedrich, D. M.; Barbara, P.

F. J. Phys. Chem. 1983, 87, 1125.

(9) Chou, P.; McMorrow, D.; Aartsma, T. J.; Kasha, M. J. Phys. Chem. $1984,88,4596$.

(10) Dzugan, T. P.; Schmidt, J.; Aartsma, T. J. Chem. Phys. Lett. 1986. 127,336 .

(11) Woolfe, G. J.; Thistlethwaite, P. J. J. Am. Chem. Soc. 1981, 103, 6916.

(12) Dick, B.; Ernsting, N. P. J. Phys. Chem. 1987, 9/, 4261

(13) Ernsting, N. P.: Dick, B. Chem. Phys. 1989, 136, 181.

(14) Rullière, C.; Declemy, A. Chem. Phys. Lett. 1987, 134, 64.

(15) Itoh, M.; Tokumura, K.; Tanimoto, Y.; Okada, Y.; Takeuchi, H.; Obi, K.; Tanaka, I. J. Am. Chem. Soc, 1982, 104, 4146.

(16) Strandjord, A. J. G.; Barbara, P. F. Chem. Phys. Lett. 1983, 98, 21.

(17) Strandjord, A. J. G.; Barbara, P. F. J. Phys. Chem. 1985, 89, 2355.

(18) McMorrow, D.; Kasha, M. J. Am. Chem. Soc. 1983, 105, 5133.

(19) McMorrow, D.; Kasha, M. J. Phys. Chem. 1984, 88, 2235. assignment is in complete agreement with that given in the experimental papers. ${ }^{1-4}$ The second transient species has been previously assigned to the tautomer ground state. ${ }^{1-4}$ Brewer et al. ${ }^{4}$ also considered a possible assignment of this transient species to the tautomer triplet state. To explain the results of the two-step laser excitation (TSLE) experiments, they proposed a decay mechanism involving a reverse intersystem crossing (ISC) step, $T_{n}{ }^{\prime} m \rightarrow S_{1}^{\prime}$. Since the quantum yield of this step had to be much higher than usually observed for this type of process, Brewer et al. remained skeptical with respect to this interpretation. Our calculations show that in the case of 3-HF the mechanism proposed by Brewer et al. is indeed reasonable.

\section{Method of Calculation}

In order to obtain the relative ordering of singlet and triplet levels of both tautomer forms of 3-HF, AM1 calculations on the geometries and heat of formation of the two electronic ground states were combined with INDO/S calculations of the excitation energies and oscillator strengths.

The AM1 method basically consists of an improved parametrization for the MNDO Hamiltonian, which has been optimized to reproduce a large set of ground-state data of organic molecules..$^{20}$ In particular, the description of hydrogen bonds has been improved as compared to the original MNDO method. ${ }^{21}$ The original MNDOC program was used, and the new parameters were implemented. From the X-ray structure analysis ${ }^{22}$ it is known that $3-\mathrm{HF}$ in the ground state of its normal form consists of two planar fragments, namely, the phenyl group and 3-hydroxychromone. We assume that both fragments remain planar in the tautomer form. The optimized geometrical parameters were all bond lengths and bond angles in the hydroxychromone part of $3-\mathrm{HF}$, all bond lengths and angles between carbon atoms in the phenyl ring, and the dihedral angle between these two parts of the molecule.

The CNDO/S method has found wide application for the calculation of excitation energies of organic compounds. ${ }^{23,24}$ Unfortunately, in the original version of this method different parametrizations are used to calculate singlet and triplet states. It could be shown, however, that a single parameter set can be used when the electron correlation is not accounted for by parametrization but by the inclusion of doubly excited configurations. ${ }^{25}$ In this case a balanced description of singlet and triplet states is obtained when the Pariser formula is used for the electron repulsion integrals. Our INDO-SDCI procedure uses 200 singly and doubly excited configurations for singlets and 300 configurations for triplets. ${ }^{26}$ The one-center integrals of the INDO Hamiltonian have been added to the original CNDO/S parameters to account for the splitting of singlet and triplet $n \pi^{*}$ states. As in previous calculations, ${ }^{25}$ the resonance integral for carbon atoms has been changed to $\beta_{C}=-16 \mathrm{eV}$. This corrects to some extent the overestimation of transition energies due to the lowering of the ground state by the doubly excited configurations.

\section{Results}

Geometry Optimization of Electronic Ground States. The calculated heat of formation is $-147.65 \mathrm{~kJ} / \mathrm{mol}$ for the ground state $S_{0}$ of the normal form and $-45.48 \mathrm{~kJ} / \mathrm{mol}$ for the ground state $\mathrm{S}_{0}{ }^{\prime}$ of the tautomer form. The optimized geometrical parameters of these two states are summarized in Figure 1. The corresponding data from the $\mathrm{X}$-ray analysis of $\mathrm{S}_{0}$ are also shown. A comparison reveals very good agreement between the calculated and the experimental geometry for the $S_{0}$ state. With the exception of two bonds in the phenyl substituent, all calculated bond lengths are slightly larger than the experimental values. The

(20) Dewar, M. J. S.; Zoebisch, E. G.; Healy, E. F.; Stewart, J. J. P. J. Am. Chem. Soc. 1985, 107, 3902 .

(21) Dewar, M. J. S.; Thiel, W. J. Am. Chem. Soc. 1977, 99, 4899.

(22) Etter, M. C.; Urbańczyk-Lipkowska, Z.; Baer, S.; Barbara, P. F. J. Mol. Struct. 1986, 144, 155 .

(23) Ellis, R. L.; Jaffe, H. H. J. Mol. Spectrosc. 1974, 50, 474

(24) Ellis, R. L.; Kuehnlenz, G.; Jaffé, H. H. Theor. Chim. Acta 1972, 26, 131 .

(25) Dick, B.; Nickel, B. Chem. Phys. 1983, 78, 1

(26) Dick, B.; Hohlneicher, G. Theor. Chim. Acta 1979, 53, 221. 


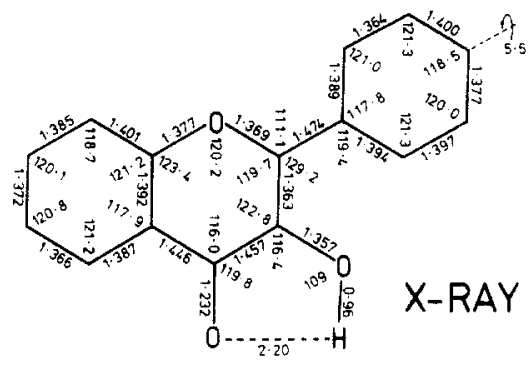

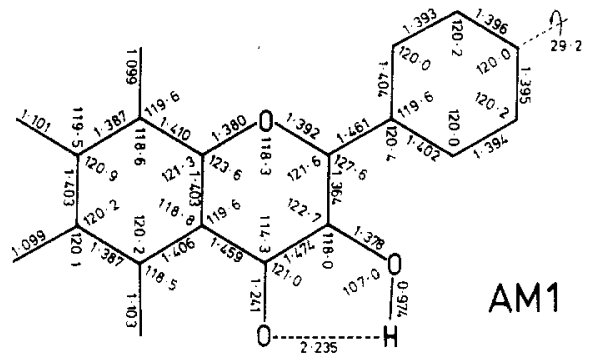

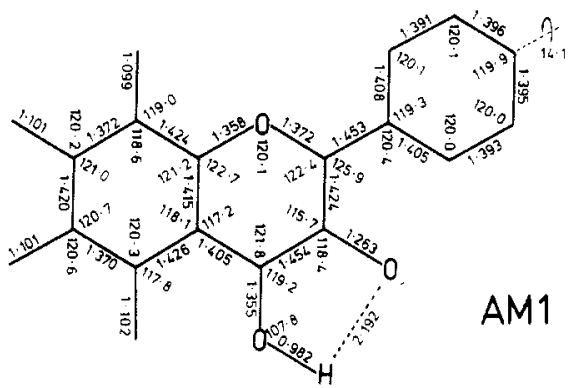

Figure 1. Bond lengths (in angstroms) and bond angles (in degrees) for the electronic ground states of 3-hydroxyflavone: (top) X-ray structure of the normal form, (middle) AM1 optimized geometry of the normal form, (bottom) AMI optimized geometry of the tautomer form.

root-mean-square deviation for the bond lengths between nonhydrogen atoms is $0.016 \AA$. It should be kept in mind that the AMI method has been optimized to reproduce geometries of isolated molecules. The crystal packing might lead to systematically smaller bond lengths. The calculated dihedral angle between the phenyl ring and the hydoxychromone part is $29.2^{\circ}$; the experimental value is $5.5^{\circ}$. It is known that the intermolecular forces in the crystal can strongly reduce dihedral angles of this kind. For example, the molecule biphenyl has a planar structure in the crystal, whereas the dihedral angle is $\approx 45^{\circ}$ in the vapor phase. ${ }^{27}$ The calculated dihedral angle of $29.2^{\circ}$ is thus reasonable for the 3-HF molecule in solution. (It should be mentioned that much larger deviations from the experimental geometry are found with the MNDO method in its original parametrization. In this case a dihedral angle of almost $90^{\circ}$ between both molecular parts is calculated.)

The calculated geometry of the $\mathrm{S}_{0}{ }^{\prime}$ state shows that the whole molecular frame is rearranged upon intramolecular proton transfer. The largest change occurs for the $\mathrm{C}-\mathrm{O}$ bonds of the keto and the hydroxy group. After proton transfer the $\mathrm{C}_{3}-\mathrm{O}$ bond shrinks by $0.115 \AA$, whereas the $\mathrm{C}_{4}-\mathrm{O}$ bond length increases by $0.114 \AA$ (See Figure 2 for the numbering of the atoms.) The single- and double-bond characters of these two bonds are thus reversed. The double-bond character of the $\mathrm{C}_{2}-\mathrm{C}_{3}$ bond is strongly reduced, and that of the $\mathrm{C}_{4}-\mathrm{C}_{4 \times}$ bond strongly increases. The $\mathrm{C}-\mathrm{O}$ bond lengths of the ether group become slightly shorter, and the anellated benzene ring exhibits a small tendency toward localization of the double bonds at the $C_{5}-C_{6}$ and $C_{7}-C_{8}$ bonds. All these observations could be interpreted as arising from an admixture of the

(27) Almenningen, A.; Bastiansen, O.; Fernholt, L.; Cyvin, B. N.; Cyvin, S. J.: Samdal, S. J. Mol. Struct. 1985, 128, 59.<smiles>O=c1oc(O)c(O)c(=O)c2ccccc12</smiles>

a<smiles></smiles>

C<smiles></smiles>

b<smiles></smiles>

d
Figure 2. Numbering scheme of atoms in 3-hydroxyflavone in its normal form (a) and some resonance structures of the tautomer form (b-d).<smiles>O=c1c(O)c(-c2ccccc2)oc2ccccc12</smiles><smiles></smiles>

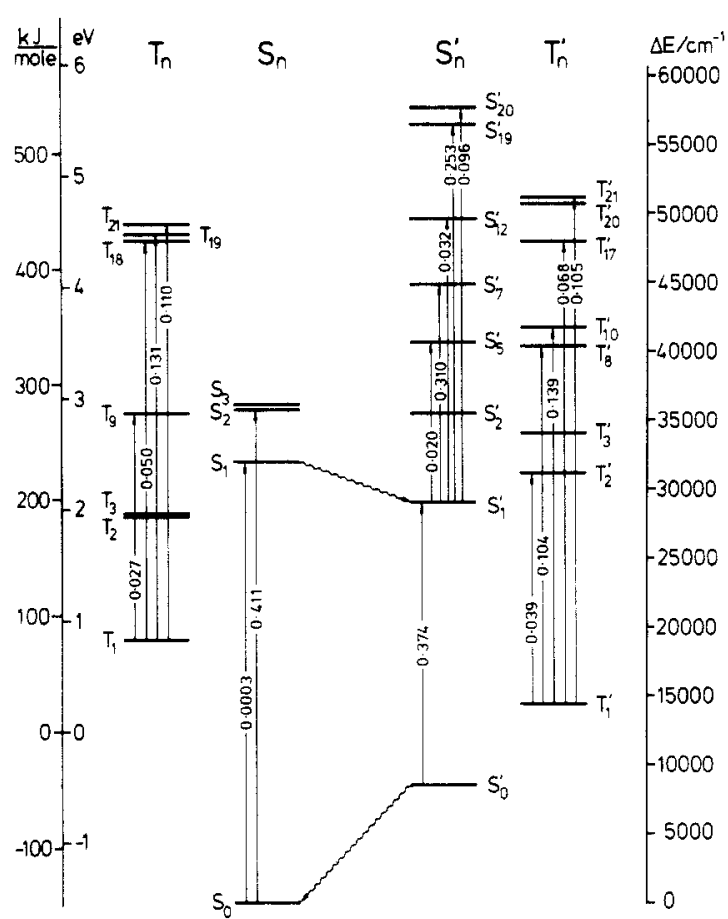

Figure 3. Energy level diagram of the calculated singlet and triplet states of the normal form of $3-\mathrm{HF}$ (left) and its tautomer form (right). Vertical arrows indicate radiative transitions, and the numbers are the calculated oscillator strengths. The left energy scale refers to the heat of formation of the AM 1 method; the right scale is relative to the electronic ground state $\mathrm{S}_{0}$.

resonance structures $c$ and $d$ in Figure 2. Whereas the bond lengths and angles in the phenyl ring show almost no change due to proton transfer, the bond connecting it to the 3-hydroxychromone becomes slightly shorter and the dihedral angle reduces to $14.1^{\circ}$, indicating an increase of the conjugative interaction between both parts of the molecule.

Excited States and Spectra. The energy level diagram of the calculated singlet and triplet states of the two tautomeric forms of $3-\mathrm{HF}$ is shown in Figure 3. The vertical arrows indicate radiative transitions, and the numbers are calculated oscillator strengths. The intramolecular-proton-transfer reaction is initiated by excitation of the normal form of 3-HF to its first excited singlet state $S_{1}$. In the calculation based on the $A M 1$ geometry the $S_{1}$ state is of $n \pi^{*}$ character, whereas the experimental extinction coefficient indicates $\pi \pi^{*}$ character for this state. ${ }^{28,29}$ The cor-

(28) Frolov, Yu. L.; Sapozhnikov, Yu. M.; Barer, S. S.; Sherstyannikova, L. V. Izv. Akad. Nauk SSSR, Ser. Khim. 1974, 1294.

(29) Remko, M.; Polčin, J. Collect. Czech. Chem. Commun. 1980, 45, 201. 
responding calculated transition is $S_{0} \rightarrow S_{2}$ with an oscillator strength of $f=0.411$. In calculations with slightly different input geometries the states $S_{1}$ and $S_{2}$ are almost degenerate. In the flavone molecule without the 3-hydroxy group the lowest excited singlet state is indeed of $n \pi^{*}$ character, ${ }^{30}$ and the same seems to be true for the 3-methoxyflavone molecule. ${ }^{3}$ This $n \pi^{*}$ state should thus be close to the $\pi \pi^{*}$ state in 3-HF.

Through ESIPT the excited-state population in $S_{1}$ is quickly transferred to $S_{1}{ }^{\prime}$. The calculated fluorescence transition $S_{1}{ }^{\prime} \rightarrow$ $S_{0}{ }^{\prime}$ lies at $20500 \mathrm{~cm}^{-1}(f=0.374)$, in good agreement with the observed maximum at $19000 \mathrm{~cm}^{-1}$. The strongest transitions of the transient absorption spectrum $\mathrm{S}_{1}{ }^{\prime} \rightarrow \mathrm{S}_{n}{ }^{\prime}$ are calculated to be $S_{1}{ }^{\prime} \rightarrow S_{7}{ }^{\prime}\left(15700 \mathrm{~cm}^{-1}, f=0.310\right)$ and $S_{1}{ }^{\prime} \rightarrow S_{19}{ }^{\prime}\left(27300 \mathrm{~cm}^{-1}\right.$, $f=0.253$ ). The first transition is in good agreement with the transient absorption observed by Rulliêre and Declêmy at 16500 $\mathrm{cm}^{-1} .^{14}$ The transient absorption with 4-ns decay time observed at $22700 \mathrm{~cm}^{-1}$ in ref 10 could be assigned to the second transition.

All $\mathrm{S}_{0}{ }^{\prime} \rightarrow \mathrm{S}_{n}{ }^{\prime}$ transitions in the visible spectral range are calculated to have low oscillator strengths, except the reverse of the fluorescence transition, $S_{0}{ }^{\prime} \rightarrow S_{1}{ }^{\prime}$. The next transition with reasonable intensity is $S_{0}^{\prime} \rightarrow S_{12}{ }^{\prime}\left(41000 \mathrm{~cm}^{-1}, f=0.210\right)$. No transient absorption measurements have been performed in this spectral range which already strongly overlaps with the absorption spectrum of the normal form of 3-HF. There exists also strong evidence that the $S_{0}^{\prime}$ state has a very short lifetime. When $3-\mathrm{HF}$ is used as a laser dye, no reabsorption due to buildup of population in $S_{0}{ }^{\prime}$ could be observed, which would lead to an unsymmetric gain profile., ${ }^{9,31}$ It is concluded that proton transfer back to the normal ground state $S_{0}$ is very rapid. Also, in an attempt to populate the $S_{0}^{\prime}$ state through stimulated emission pumping, no transient absorption could be seen with a time resolution of 30 ps. ${ }^{10}$ Finally, even at temperatures below $15 \mathrm{~K}$ the tautomer ground state could not be trapped in argon matrices. 12,32,33 Hence, the back-reaction $S_{0}^{\prime} u_{n} \rightarrow S_{0}$ cannot be a thermally activated process and should be very fast at room temperature. It is, therefore, extremely unlikely that the transient species with $\approx 14-\mu \mathrm{s}$ decay time observed in refs 3 and 4 is the tautomer ground state $\mathrm{S}_{0}{ }^{\prime}$.

The possibility should thus be examined that both long-lived transient absorptions are due to triplet species. The most likely candidates are $T_{1}$ and $T_{1}{ }^{\prime}$. The experimental papers agree in the assignment of the transient species with absorption peak at 25300 $\mathrm{cm}^{-1}$ and 7.2- $\mu$ s decay time to a triplet state. The choice of $T_{1}$, however, should be reexamined since it was mainly based on the assignment of the other species to the tautomer ground state. The calculated absorption spectrum of $T_{1}$ has three reasonably intense transitions in the near-UV region: $\mathrm{T}_{1} \rightarrow \mathrm{T}_{18}\left(28900 \mathrm{~cm}^{-1}, f=\right.$ $0.050), \mathrm{T}_{1} \rightarrow \mathrm{T}_{19}\left(29400 \mathrm{~cm}^{-1}, f=0.131\right)$, and $\mathrm{T}_{1} \rightarrow \mathrm{T}_{21}(30100$ $\left.\mathrm{cm}^{-1}, f=0.11\right)$. The strongest low-lying transitions starting from $\mathbf{T}_{1}{ }^{\prime}$ occur at lower energies, namely, $\mathbf{T}_{1}{ }^{\prime} \rightarrow \mathrm{T}_{2}^{\prime}\left(16700 \mathrm{~cm}^{-1}, f\right.$ $=0.039), \mathrm{T}_{1}^{\prime} \rightarrow \mathrm{T}_{8}^{\prime}\left(25900 \mathrm{~cm}^{-1}, f=0.104\right)$, and $\mathrm{T}_{1}^{\prime} \rightarrow \mathrm{T}_{10}{ }^{\prime}$ $\left(27400 \mathrm{~cm}^{-1}, f=0.139\right)$. The relative magnitude of the transition energies suggests to assign the transient absorption at $25300 \mathrm{~cm}^{-1}$ to the transitions $T_{1} \rightarrow\left(T_{18}, T_{19}, T_{21}\right)$ and the transient absorption at $22900 \mathrm{~cm}^{-1}$ to the transitions $\mathrm{T}_{1}^{\prime} \rightarrow\left(\mathrm{T}_{8}{ }^{\prime}, \mathrm{T}_{10}{ }^{\prime}\right)$. The assignment of the transient species with $7.2-\mu \mathrm{s}$ decay time to $T_{1}$ then reinforces the choice made in the experimental papers. If this assignment is accepted, the calculated transition energies overestimate the experimental ones by $\approx 3500 \mathrm{~cm}^{-1}$ in both cases. The INDO/S method frequently overestimates transition energies, especially for large molecules for which the selected configuration basis consists only of a small fraction of the possible configurations. It should also be kept in mind that the triplet-triplet absorption spectra have been calculated for the geometries of the electronic ground states. One reason for the overestimated transition energies could be that the actual geometries of the states $T_{1}$ and $T_{1}{ }^{\prime}$ are slightly different.

(30) Pownall, H. J. Spectrochim. Acta, Part A 1974, 30, 953.

(31) Chou, P.: Aartsma, T. J. J. Phys. Chem. 1986, 90,721

(32) Brucker, G. A.; Kelley, D. F. J. Phys. Chem. 1987, 91, 2862.

(33) Brewer, W. E.; Studer, S. L.; Chou, P.-T.; Orton, E. Chem. Phys. Lett. 1989, 158,345 .

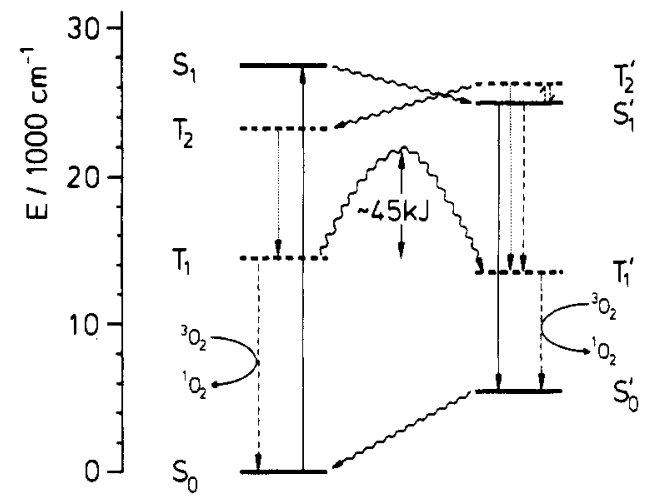

Figure 4. Energy level diagram of the lowest electronic states of the normal form of 3-HF (left) and its tautomer form (right). The various photophysical processes are indicated by straight arrows (radiative transitions), curled arrows (proton transfer), broken arrows (ISC), and dotted arrows (IC).

It is very interesting to note the large energy difference calculated between $T_{2}^{\prime}$ and $T_{1}^{\prime}$. Its size of $16700 \mathrm{~cm}^{-1}$ is quite unusual for a gap between triplet states in a large organic compound. In fact, this gap is comparable in size to the gap between $S_{0}$ and $S_{1}$ in many dye molecules. This should have important consequences for the decay properties of the $T_{2}{ }^{\prime}$ state, which should be similar to those of the $S_{1}$ state in dye molecules. This means that the time constant of the internal conversion $T_{2}{ }^{\prime}$ m $\rightarrow T_{1}{ }^{\prime}$ could be well in the nanosecond range, allowing ample time for a reasonable fraction of molecules to decay to the $S_{1}{ }^{\prime}$ state by ISC. Furthermore, one might observe fluorescence light in the visible spectral range from the $T_{2}{ }^{\prime} \rightarrow T_{1}^{\prime}$ transition.

The gap between $T_{1}{ }^{\prime}$ and $S_{0}{ }^{\prime}$ is calculated to be rather small, namely, $5800 \mathrm{~cm}^{-1}$. This energy is only slightly smaller than the amount needed to produce singlet oxygen in an oxygen quenching reaction. The calculated energy gap between $T_{1}$ and $S_{0}$ of 19000 $\mathrm{cm}^{-1}$ is sufficiently large for the production of singlet oxygen. The phosphorescence $T_{1} \rightarrow S_{0}$ should occur in the visible spectral range and might be observable at low temperatures.

\section{Discussion}

The assignments made in the preceding section have important consequences for the photophysics of $3-\mathrm{HF}$. These will be discussed in the following based on the level diagram shown in Figure 4 which has been composed to agree with the experimentally known as well as the calculated energy data, keeping in mind that the latter are only accurate to within $\pm 2000 \mathrm{~cm}^{-1}$. The experimental $S_{0} \rightarrow S_{1}$ excitation energy is $\approx 27500 \mathrm{~cm}^{-1}$; that of the fluorescence transition $S_{1}^{\prime} \rightarrow S_{0}^{\prime}$ is $\approx 19500 \mathrm{~cm}^{-1}$. The Stokes shift of $\approx 8000 \mathrm{~cm}^{-1}$ is the sum of the energy shifts of the ground state and the first excited singlet state. These shifts are not known individually, and we assume that they are similar in size. This is in line with the calculated energy values and with the experimental observation that the proton-transfer reactions in both states are irreversible.

The gap between $S_{0}{ }^{\prime}$ and $T_{1}{ }^{\prime}$ in Figure 4 is $\approx 8000 \mathrm{~cm}^{-1}$, slightly larger than the calculated value of $5800 \mathrm{~cm}^{-1}$. This accounts for the fact that quenching of $T_{1}{ }^{\prime}$ with oxygen produces singlet oxygen, since the observed quantum yield of photooxidation products is very low. ${ }^{4}$ If the gap between $T_{1}{ }^{\prime}$ and $S_{0}{ }^{\prime}$ were too small to provide the energy needed for the production of singlet oxygen, a quenching reaction $T_{1}^{\prime} u m \rightarrow S_{0}$ would have to be postulated. Such a process involving simultaneous ISC and proton transfer seems to be rather unlikely.

If we assume that $T_{1}$ is slightly lower than calculated, it will be almost isoenergetic with $T_{1}^{\prime}$. In this case a barrier to ESIPT on the lowest triplet surface could exist. Such a situation has been previously observed in the molecule 2-(2-hydroxyphenyl)benzoxazole. $^{34}$ A barrier must be postulated to account for the long

(34) Grellmann, K. H.; Mordziñski, A.; Heinrich, A. Chem. Phys. 1989, 136,201 . 
lifetime of both triplet states. If we assume that the decay of $T_{1}$ is mainly due to proton transfer, the decay time of $7.2 \mu \mathrm{s}$ at room temperature corresponds to an energy barrier between 39 and 50 $\mathrm{kJ} / \mathrm{mol}$ for a reaction with a frequency factor between $10^{12}$ and $10^{14} \mathrm{~s}^{-1}$. To check this prediction, we propose to populate $T_{1}$ with a triplet sensitizer. In this case $T_{1}{ }^{\prime}$ should not initially be populated, and the transient absorption at $22900 \mathrm{~cm}^{-1}$ should grow in with the decay time of $T_{1}$.

A question that remains to be answered concerns the pathway of population of $T_{1}$ after excitation of 3 -HF to the $S_{1}$ state. The direct way would be ISC $S_{1} \rightarrow T_{1}$. In this case ISC must be very fast to compete with ESIPT. Fast ISC on a picosecond time scale has indeed been observed for several aromatic ketones, ${ }^{35,36}$ but it is questionable whether these processes are really fast enough to compete with ESIPT. Alternatively, $T_{1}$ could be populated through ISC combined with reverse proton transfer, $S_{1}{ }^{\prime}$ m $\rightarrow T_{1}$. The probability of such a simultaneous process should, however, be rather low. An interesting decay path involves the $T_{2}$ surface. According to the calculations, $T_{2}{ }^{\prime}$ is slightly above $S_{1}{ }^{\prime}$, but the reversed ordering is also in accordance with the accuracy of the calculations. Hence, $T_{2}^{\prime}$ could be populated via ISC from $S_{1}{ }^{\prime}$. The calculations also predict that $T_{2}$ is below $T_{2}^{\prime} ;$ thus, proton back-transfer should occur on the $T_{2}$ surface. Subsequent internal conversion (IC) $T_{2} \rightarrow T_{1}$ then leads to population in $T_{1}$. If $T_{1}$ is mainly populated by direct ISC, the transient absorption at $25300 \mathrm{~cm}^{-1}$ should rise with the same rise time as the tautomer fluorescence. For the two other processes the bottleneck will be $\mathrm{S}_{1}{ }^{\prime}$, and the transient absorption should rise with the decay time of the tautomer fluorescence. If the path involving the $\mathrm{T}_{2}{ }^{\prime}$ state is active, excitation of $T_{1}{ }^{\prime}$ should lead to an increase of the transient absorption assigned to $T_{1}$.

The large energy gap between $T_{1}^{\prime}$ and $T_{2}^{\prime}$ has important consequences for the explanation of the two-step laser excitation (TSLE) experiments. ${ }^{3,4}$ Excitation $\mathrm{T}_{1}{ }^{\prime} \rightarrow \mathrm{T}_{n}{ }^{\prime}$ will, after IC $\mathrm{T}_{n}{ }^{\prime}$ um $\rightarrow T_{2}^{\prime}$. lead to population in $T_{2}^{\prime}$. This population can decay in three ways: internal conversion $T_{2}^{\prime} m \rightarrow T_{1}^{\prime}$, reverse proton transfer $T_{2}^{\prime} m \rightarrow T_{2}$, and reverse ISC $T_{2}^{\prime} T_{m} \rightarrow S_{1}^{\prime}$. Internal conversion should be slow due to the large energy gap. The reverse proton transfer has been discussed above as a possible path for the population of $T_{1}$. The molecules produced in $S_{1}{ }^{\prime}$ via reverse ISC will lead to the typical tautomer fluorescence $S_{1}{ }^{\prime} \rightarrow S_{0}{ }^{\prime}$. The apparent quantum yield of tautomer fluorescence following excitation of the $14.2-\mu \mathrm{s}$ species was estimated to be 0.013 in ref 4 , compared to 0.36 for the population of $S_{1}{ }^{\prime}$ via proton transfer. If the latter value is interpreted as the intrinsic quantum yield of tautomer fluorescence at room temperature, an estimated yield of 0.036 is calculated for reverse ISC $T_{2}{ }^{\prime} m \rightarrow S_{1}{ }^{\prime}$.

A large yield of reverse ISC has previously been found for other molecules with a large energy gap between $T_{1}$ and $T_{2}$. In 9. phenylanthracene (9-PA) and 9-methylanthracene (9-MA) these yields are $8.3 \times 10^{-5}$ and $6.1 \times 10^{-5} .37$ The rate constant for the reverse ISC is $4.9 \times 10^{9} \mathrm{~s}^{-1}$ in 9-MA. ${ }^{38}$ In 9,10-dibromoanthracene an even larger yield of 0.19 has been found ${ }^{39}$ The energy gap between $T_{1}$ and $T_{2}$ is $\approx 11000 \mathrm{~cm}^{-1}$ in these molecules. ${ }^{38}$ In $3-H F$ the gap is probably larger, leading to an even slower decay

(35) Greene, B. I.; Hochstrasser, R. M.; Weisman, R. B. J. Chem. Phys. 1979. 70,1247 .

(36) Choi, K. J.; Hallidy, L. A.; Topp, M. R. In Picosecond Phenomena II; Hochstrasser, R. M., Kaiser, W., Shank, C. V., Eds.; Springer: Berlin, 1980; p 131 .

(37) Kobayashi, S.; Kikuchi, K.; Kokubun, H. Chem. Phys. Lett. 1976, 42, 494.

(38) Fukumura, H.; Kikuchi, K.; Koike, K.; Kokubun, H. Photochem. Photobiol. A 1988, 42, 283.

(39) Kikuchi, K.; Fukumura, H.; Kokubun, H. Chem. Phys. Lett. 1986, 123,226 . of $T_{2}$ through internal conversion. The presence of a keto group might further speed up the reverse ISC. But the dominant decay process of $\mathrm{T}_{2}{ }^{\prime}$ might well be the reverse proton transfer.

An order-of-magnitude estimate of the rate constants involved in the decays of the states $S_{1}{ }^{\prime}$ and $T_{2}{ }^{\prime}$ can be based on the following arguments. Let us denote the rate constants of forward and backward ISC between $S_{1}{ }^{\prime}$ and $\mathrm{T}_{2}{ }^{\prime}$ by $k_{\mathrm{ST}}$ and $k_{\mathrm{TS}}$ and the rate constants of the decays of $S_{1}^{\prime}$ and $T_{2}^{\prime}$ to other states by $k_{S}$ and $k_{\mathrm{T}}$, respectively. The ratio of the two ISC rate constants depends on the energy difference $\Delta E=E\left(\mathrm{~T}_{2}{ }^{\prime}\right)-E\left(\mathrm{~S}_{1}{ }^{\prime}\right)$, through

$$
\frac{k_{\mathrm{ST}}}{k_{\mathrm{TS}}}=3 \exp \left(-\frac{\Delta E}{k_{\mathrm{B}} T}\right)
$$

The ratio of the quantum yield of TSLE fluorescence and the normally excited tautomer fluorescence within this model is

$$
\frac{\Phi_{\mathrm{F}}^{\mathrm{TSLE}}}{\Phi_{\mathrm{F}}}=\frac{k_{\mathrm{TS}}}{k_{\mathrm{TS}}+k_{\mathrm{T}}}
$$

The experimental value of this ratio is 0.036 , leading to $k_{\mathrm{T}} / k_{\mathrm{TS}}$ $=26.8$. Thus, the decay of $T_{2}{ }^{\prime}$ to $T_{1}{ }^{\prime}$ and $T_{2}$ is much faster than reverse ISC. Hence, the total decay rate constant of $S_{1}{ }^{\prime}$ is in good approximation given by the sum of $k_{\mathrm{S}}$ and $k_{\mathrm{ST}}$. Its value at room temperature is $2.5 \times 10^{8} \mathrm{~s}^{-1}$. If we assume that the main contribution to $k_{S}$ is the radiative decay with a quantum yield of 0.36 , a value of $1.6 \times 10^{8} \mathrm{~s}^{-1}$ is found for $k_{\mathrm{ST}}$. If $\mathrm{S}_{1}{ }^{\prime}$ and $\mathrm{T}_{2}^{\prime}$ are isoenergetic, eq 1 leads to $k_{\mathrm{TS}}=5.3 \times 10^{7} \mathrm{~s}^{-1}$. Equation 2 then yields $k_{\mathrm{T}}=1.4 \times 10^{9} \mathrm{~s}^{-1}$, corresponding to a lifetime of $700 \mathrm{ps}$ for $\mathrm{T}_{2}{ }^{\prime}$. With $\Delta E=500 \mathrm{~cm}^{-1}$ one obtains $k_{\mathrm{TS}}=6.0 \times 10^{8} \mathrm{~s}^{-1}$, $k_{\mathrm{T}}=1.6 \times 10^{10} \mathrm{~s}^{-1}$, and a lifetime of $\mathrm{T}_{2}^{\prime}$ of $62 \mathrm{ps}$.

The relative ordering of the states $S_{1}^{\prime}$ and $T_{2}^{\prime}$ could experimentally be verified through measurement of the temperature dependence of the yield of $T_{1}$ after normal excitation and the yield of reverse ISC after two-step laser excitation. If $\mathrm{T}_{2}^{\prime}$ is slightly higher than $S_{1}{ }^{\prime}$ as suggested by the calculations, the forward ISC step would be thermally activated. With decreasing temperature the decay of $S_{1}{ }^{\prime}$ through this channel should become slower, leading to a lower quantum yield of $T_{1}$ and a longer lifetime of $\mathrm{S}_{1}{ }^{\prime}$. The latter fact is indeed observed but could also be due to a different thermally activated radiationless decay channel. If $T_{2}{ }^{\prime}$ is below $S_{1}{ }^{\prime}$, the reverse ISC step should be thermally activated. Then the apparent quantum yield of tautomer fluorescence after TSLE relative to that after direct excitation should decrease with decreasing temperature.

\section{Conclusions}

The comparison of our calculations with the experimental data known from the literature leads us to the following conclusions:

1. The two long-lived transient species with decay times of 7.2 and $14.2 \mu \mathrm{s}$ are assigned to the states $T_{1}$ and $T_{1}{ }^{\prime}$.

2. As a consequence of this assignment, a substantial barrier to proton transfer must exist along the potential curve of the lowest triplet state.

3. An unusually long lifetime of several picoseconds is proposed for the second excited triplet state $\mathrm{T}_{2}^{\prime}$ of the tautomer. This fact allows ISC to the $S_{1}{ }^{\prime}$ state to compete with the decay of $T_{2}{ }^{\prime}$ to $T_{1}{ }^{\prime}$ and reverse proton transfer to $T_{2}$. The transition $T_{2}^{\prime} \rightarrow T_{1}{ }^{\prime}$ could also lead to an observable triplet-triplet fluorescence.

Acknowledgment. The author acknowledges generous support of this work by Prof. F. P. Schäfer, as well as stimulating discussions with Drs. K. H. Grellmann and B. Nickel. This work has been supported by the Deutsche Forschungsgemeinschaft through the Leibniz-Prize program and under the project SFB 93/D8. 